\title{
OBESITY
}

\section{Methylation a consequence not a cause}

Alterations in DNA methylation are a consequence of increased adiposity according to new data published in Nature. These epigenomic changes might also predict the occurrence of type 2 diabetes mellitus (T2DM) independently of traditional risk factors.

The investigators used samples from 5,387 individuals and identified 187 DNA methylation sites that were associated with BMI. The team also isolated adipocytes from 24 individuals with obesity $\left(\mathrm{BMI}>40 \mathrm{~kg} / \mathrm{m}^{2}\right)$ and 24 individuals of normal weight, and found that 6 of the 187 markers were highly correlated with obesity $\left(P<2.7 \times 10^{-4}\right)$ and a further 108 also had a weak, but significant, association $(P=0.04)$.

To assess the role of these markers, the team first used genetic association analyses and found that methylation changes seem to be a consequence of increased BMI, rather than a cause. These changes also had functional consequences; on average, in 38 genes, a 5\% change in methylation led to a $7 \%$ alteration in gene expression. The products of many of these genes are involved in lipid metabolism, substrate transport and inflammation.

Finally, 62 markers were associated with the development of T2DM. The strongest association was with a marker in the $A B C G 1$ locus, which is required for insulin secretion and $\beta$-cell function. Importantly, this association was independent of conventional T2DM risk factors such as adiposity and glucose tolerance.

The authors hope their results will be applicable in a personalized medicine setting and for risk stratification of patients who might go on to develop more serious metabolic diseases.

Tim Geach

ORIGINAL ARTICLE Wahl, S. et al. Epigenomewide association study of body mass index, and the adverse outcomes of adiposity. Nature http://dx.doi.org/10.1038/nature20784 (2016) 